\title{
A time-frequency analysis of the Canadian macroeconomy and the yield curve
}

\author{
Mustapha Olalekan Ojo* Luís Aguiar-Conraria ${ }^{\dagger} \quad$ Maria Joana Soares ${ }^{\ddagger}$
}

September 24, 2018

\begin{abstract}
We use wavelet analysis to study the relationship between the yield curve and macroeconomic indicators in Canada. We rely on the Nelson-Siegel approach to model the zero coupon yield curve, and use the Kalman filter to estimate its time-varying factors: the level, the slope and the curvature. Apart from the bidirectional yield-macro relation, the paper broadens the existing literature by exploring the link between the monetary policy and the yield curve.

We reached several conclusions. First, the monetary policy variable, the bank rate, affects mainly short-run interest rates. Arguably, the main driver for economic activity is the long run interest rate (instead of the short run), suggesting that monetary policy is mostly ineffective. Second, we concluded that concerning the inflation rate, the Bank of Canada is very proactive. Third, regarding the unemployment rate, we found that both the slope and the curvature are leading indicators for the long run evolution of unemployment. Finally, our results suggest that the industrial production index leads the yield curve factors and not the other way.
\end{abstract}

Keywords: Term structure; Yield curve; Macroeconomic variables;Wavelet Power Spectrum; Wavelet Coherency; Wavelet Phase Difference.

JEL classification: E43; E44; C32; C49;

This work was carried out within the funding with COMPETE reference number POCI01-0145-FEDER-006683, with the FCT/MEC's (Fundação para a Ciência e a Tecnologia,

\footnotetext{
*Economics Department, University of Minho. E-mail: mustaphaojo@gmail.com

${ }^{\dagger}$ Corresponding author. NIPE and Economics Department, University of Minho. E-mail: lfaguiar@eeg.uminho.pt. Telephone: (+351) 964108697

${ }^{\ddagger}$ NIPE and Department of Mathematics and Applications, University of Minho. E-mail: jsoares@math.uminho.pt
} 
I.P.) financial support through national funding and by the ERDF through the Operational Programme on "Competitiveness and Internationalization - COMPETE 2020 under the PT2020 Partnership Agreement. 


\section{Introduction}

"The ability of the monetary policy to affect aggregate expenditures rests on the premise to influence market expectations regarding the future path of short-term interest rates" — Geiger (2001, p.1). However the impact of any central bank on macroeconomic dynamics depends on its influence on financial market prices, particularly, its grip on the long-term interest rate, which governs the level of credit demand and consequently, expenditures. The typical hypothesis is that expectations connect the short and long run interest rates, with the yield curve representing the pivot between the aggregate demand and monetary policy, offering the central bank valuable information on private market expectations (Geiger, 2011). Such vital influence has fueled a proliferation of yield curve models.

While the yield curve modeling is evolving — see Diebold and Rudebusch (2013) for a review —, efforts have been made to explore the unidirectional and bidirectional links between macroeconomic variables and the yield curve. Such efforts deployed mostly traditional econometrics techniques (see Ang and Piazzesi, 2003; Wu, 2002; Estrella and Hardouvelis, 1991; Benati and Goodhart, 2008), with few novel approaches (see Aguiar-Conraria, Martins and Soares, 2012). However, there is a paucity of literature on the role of the yield curve in the monetary policy space. Such investigation is crucial for countries like Canada, since its central bank uses an interest rate measure - Target for the Overnight Rate - as a monetary transmission mechanism. This paper fills the void by, not only exploring the bidirectional macro-yield link, but also evaluating the relationship between the Canadian yield curve and its monetary policy. The paper equally deepens the literature by adopting the wavelet approach. The ability of wavelet tools to capture the high irregularity in the financial data through its decomposition into both time and frequency domain influences its choice for this study.

A wide range of empirical studies has explored the relationship between the yield curve and the macroeconomic variables. Diebold, Rudebusch and Aruoba (2006) used the NelsonSiegel model to explore the macroeconomic-yield link and found that the inflation and real activity highly correlate with level and slope factors respectively, but the curvature factor has 
no underlying relationship with any of the main macroeconomic variables. Evans and Marshall (2007) establish that inflation and economic activity are responsible for the huge variability in short and medium-term yields, respectively. The explanatory power of these two variables is commonly attributed to the role of monetary policy as a transmission channel of macroeconomic dynamics (Kozicki and Tinsley 2001). Rudebusch and Wu (2008) obtained a similar result, but with a more structural interpretation: level factor reflects the market underlying view about central bank's medium-term inflation targeting while slope factor captures central bank's cyclical response. Empirical studies by Kozicki and Tinsley (2001) and Dewachter and Lyrio (2006) explore the feedback from an implicit inflation target generated from the yield curve to explain the yield curve dynamics. Similarly, Taylor (1993) found that the short-end of the yield curves evolves at par with the central bank's policy instrument, which responds to changes in inflation and economic activity. Furthermore, Moneta (2005) hypothesized that a positive yield curve is associated with the future expected economic growth by investors, while a negative yield curve is associated with an expected impairment in economic growth.

To our knowledge, two papers used wavelets to explore this relation for the United States. Gallegati et al. (2014) rely on the Discrete Wavelet Transform to perform a scale-by-scale decomposition of several indicators that measure the stance of monetary policy variables, including the yield curve slope, and GDP. Similarly, Aguiar-Conraria, Martins and Soares (2012) relied on Continuous Wavelet Transform to explore the link between the yield curve and the macroeconomy in the United States. The main advantage of using wavelets is its utility to estimate relations that are not only time-, but also frequency-varying, providing a thorough vision of the inter-relation between the yield curve components and the macro variables that is almost impossible to obtain with purely time-domain or frequency-domain analyses.

The literature on the Canadian yield curve is scarce, and the limited available studies have widespread focus. For instance, Lange (2013) explored the link between the Canadian yield curve and its macroeconomic variables using a dynamic latent approach and found a bidirectional causality between them. Garcia and Luger (2007) equally explore this relationship using the equilibrium-based approach and incorporate a vector autoregression description of 
Canada's key macroeconomic dynamics. They found that in-sample average pricing errors from the equilibrium-based model are slightly larger than those from a relatively flexible no-arbitrage model. However, Hao and Ng (2011) investigated the ability of Canadian financial and macroeconomic variables in predicting recessions and found that government bond yield spread (akin to the yield slope) among others is a powerful predictor of recession. The study by Booth et al. (2007) focused on the drivers of provincial-Canada yield spreads and found a strong correlation between provincial yield spreads and provincial debt and deficit levels. Hejazi et al. (2000) examined the implicit determinants of the Canadian term premia and found that the conditional variances of Canadian macroeconomic variables are not significant predictors of the T-bill term structure. This finding is contrary to the evidence from the US.

While a strand of literature on the yield curve focuses on the link between the yield curve and macroeconomic variables and its predictive power, there is paltry literature on the yield curve and the monetary policy. Cook and Hahn (1989) examined the market's reaction to monetary policy actions and found a positive response to target increases at all maturities. Edelberg and Marshall (1996) found a marginal significance response of bond rates to policy shocks, but a highly significant response regarding bill rates.

The paper proceeds as follows. In section 2, we describe our modeling and estimation choices for the yield curve factors; we provide a summary of the continuous wavelet tools that we use, and present the data. In section 3, we present our main results and section 4 concludes.

\section{Methodology and Data}

\subsection{Wavelet Tools}

This is a summary of the wavelet tools that we will use in this paper. The proficient reader may skip this section without loss. Aguiar-Conraria and Soares (2014) provide a much deeper and technical description of the properties of the Continuous Wavelet Transform. For a much more intuitive overview of this rather novel technique, the reader is invited to read Aguiar-Conraria 
et al. (2012 and 2013).

Time-scale wavelets are characterized in reference to a mother wavelet, $\psi(t)$, of a real variable t. For a function to qualify as a mother wavelet it must satisfy a certain admissibility condition which, in practice, amounts to requiring that the function integrates to zero (implying that it has to be oscillatory) and has a fast decay towards zero. The mother wavelet $\psi$ provides a source function for generating daughter wavelets, $\psi_{\mathrm{t}, \mathrm{s}}$. This is achieved by translating and scaling $\psi$ by $\mathrm{t}$ and $\mathrm{s}$, respectively, to obtain:

$$
\psi_{\mathrm{t}, \mathbf{s}}(t)=\frac{1}{\sqrt{|\mathbf{s}|}} \psi\left(\frac{t-\mathrm{t}}{\mathrm{s}}\right), \mathrm{t}, \mathbf{s} \in \mathbb{R}, \mathbf{s} \neq 0
$$

Given a time series $x(t)$, its continuous wavelet transform with respect to the wavelet $\psi$ is a function of two variables, $W_{x}(\mathrm{t}, \mathrm{s})$, given by:

$$
W_{x}(\mathrm{t}, \mathrm{s})=\int_{-\infty}^{\infty} x(t) \bar{\psi}_{\mathrm{t}, \mathrm{s}}(t) d t=\frac{1}{\sqrt{|\mathbf{s}|}} \int_{-\infty}^{\infty} \bar{\psi}\left(\frac{t-\mathrm{t}}{\mathrm{s}}\right) d t
$$

with the bar denoting complex conjugate.

When the wavelet $\psi$ is a complex-valued function, the wavelet transform $W_{x}(\mathrm{t}, \mathbf{s})$ is also complex-valued and can, therefore, be expressed in polar form as $W_{x}(\mathrm{t}, \mathrm{s})=\left|W_{x}(\mathrm{t}, \mathrm{s})\right| e^{i \phi_{x}(\mathrm{t}, \mathrm{s})}, \phi_{x} \in$ $(-\pi, \pi]$. The angle (or argument) $\phi_{x}(\mathrm{t}, \mathrm{s})$ is known as the wavelet phase. For real-valued wavelet functions, the imaginary part is constantly zero and the phase is, therefore, uninformative. Hence, to obtain phase information about time-series — which will be essential to assess the lead/lag relationships between two series —, it is mandatory to make use of complex wavelets. In this paper we use one of the most popular complex wavelets available, a member of the so-called Morlet family, first introduced in Goupillaud et al. (1984):

$$
\psi_{\omega_{0}}(t)=\pi^{-\frac{1}{4}} e^{i \omega_{0} t} e^{-\frac{t^{2}}{2}}
$$

Our particular choice corresponds to considering $\omega_{0}=6$ and is dictated by the fact that, for this value of the parameter $\omega_{0}$, there is a very simple one-to-one relation between scale and 
Fourier frequency, $f \approx \frac{1}{s}$, which greatly facilitates the interpretation of the results; see e.g. Aguiar-Conraria and Soares (2013) for more details on this and other appealing properties of the Morlet wavelets.

The paper utilizes three wavelet tools — wavelet power spectrum, wavelet coherency and wavelet phase difference — that we now describe.

Remark 1 As for the wavelet transform, the wavelet power spectrum, the wavelet coherency and the wavelet phase difference are functions of the two variables $\mathrm{t}$ and $\mathbf{s}$; in what follows, for a question of simplicity, when referring to these functions, we omit the argument $(\mathrm{t}, \mathrm{s})$.

\subsubsection{Wavelet Power}

The wavelet power spectrum or scalogram is simply the square of the modulus of the wavelet transform, i.e. is given by

$$
W P S_{x}=\left|W_{x}\right|^{2}
$$

The power spectrum measures the local variance of the time-series $x(t)$ in the time-frequency domain.

In the paper, the wavelet power spectrum is used to evaluate the degree of variability across time and frequencies for the level, slope, and curvatures factors of the Canadian yield curve, as well as for each of the macroeconomic variables considered.

\subsubsection{Cross-Wavelet Power and Coherency}

The cross-wavelet transform of two time series $x(t)$ and $y(t)$, denoted by $W_{x y}$, is given by $W_{x y}=W_{x} \overline{W_{y}}$.

In analogy with the concept of coherency used in Fourier analysis, we define the complex wavelet coherency of $x(t)$ and $y(t)$, denoted by $\varrho_{x y}$ by:

$$
\varrho_{x y}=\frac{S\left(W_{x y}\right)}{\left[S\left(\left|W_{x}\right|^{2}\right) S\left(\left|W_{y}\right|^{2}\right)\right]^{1 / 2}},
$$


where $S$ denotes a smoothing operator in both time and scale; time and scale smoothing can be achieved by convolution with appropriate windows; see Cazelles et al. (2007) for details.

The absolute value of the complex wavelet coherency is called the wavelet coherency and is denoted by $R_{x y}$, i.e.

$$
R_{x y}=\frac{\left|S\left(W_{x y}\right)\right|}{\left[S\left(\left|W_{x}\right|^{2}\right) S\left(\left|W_{y}\right|^{2}\right)\right]^{1 / 2}} .
$$

Note that we have $0 \leq R_{x y} \leq 1$

\subsubsection{Wavelet Phase Difference}

While the wavelet coherency measures the relationship between each of the referenced macroeconomic variables and the latent factors, the wavelet phase difference assesses the type of relationship - phase or anti-phase - and identifies the lead and lag variables.

The phase difference between two series $x$ and $y$, denoted by $\phi_{x, y}$, is simply the angle of the complex coherency, i.e. the angular measure expressed in radians computed from

$$
\tan \phi_{x y}=\frac{\Im\left(\varrho_{x y}\right)}{\Re\left(\varrho_{x y}\right)}, \quad \phi_{x y} \in(-\pi, \pi],
$$

together with the information on the signs of the imaginary part, $\Im\left(\varrho_{x y}\right)$, and real part, $\Re\left(\varrho_{x y}\right)$, of $\varrho_{x y}$ to determine to which quadrant $\phi_{x y}$ belongs to. The obtained results should be interpreted as follows. If $\phi_{x, y}=0$, then the series are totally in phase, while if $\phi_{x, y}=\pi$, the series show a total anti-phase relationship; if $x$ lies between 0 and $\pi / 2$, then the series are in-phase, but the variable $x$ leads $y$; if $x$ is between $-\pi / 2$ and 0 , the series are also in-phase, with $y$ leading; when $\phi$ is between $-\pi$ and $-\pi / 2$ or between $\pi / 2$ and $\pi$, the series show in anti-phase relation and, in the first case, $x$ leads $y$, while in the second case, is $y$ which leads.

\subsection{Yield Curve Model Specification and Estimation}

Despite a growing body of literature on yield curve modeling, three distinct approaches are popular: no-arbitrage models, equilibrium models and parsimonious model. While the no- 
arbitrage approach perfectly fixes the yield curve at a point in time and ensures no arbitrage opportunity exist, the equilibrium approach typically uses affine models to model the dynamics of the instantaneous rates and subsequently derive yields of other maturities under various assumptions about the risk premium. The parsimonious model, popularized by Nelson and Siegel (1987), distills the yield curve into a three-dimensional parameter: level, slope, and curvature.

This study uses the parsimonious model to explore the relationship between the Canadian yield curve and the macroeconomic variables. The model is represented by:

$$
y(\tau)=\beta_{1}+\beta_{2}\left(\frac{1-e^{-\lambda \tau}}{\lambda \tau}\right)+\beta_{3}\left(\frac{1-e^{-\lambda \tau}}{\lambda \tau}-e^{-\lambda \tau}\right),
$$

where $y(\tau)$ and $\tau$ denote the zero-coupon yields and maturity respectively and $\beta_{1}, \beta_{2}$ and $\beta_{3}$ are time-varying parameters. Based on Diebold and Li (2006), the time-varying parameters capture the level, slope and curvature of the yield curve at each period, $t$. The yield can be estimated from the equation:

$$
y_{t}(\tau)=L_{t}+S_{t}\left(\frac{1-e^{-\lambda \tau}}{\lambda \tau}\right)+C_{t}\left(\frac{1-e^{-\lambda \tau}}{\lambda \tau}-e^{-\lambda \tau}\right)
$$

The role of the three components $L_{t}, S_{t}$ and $C_{t}$ becomes clear when we consider their limiting behavior with respect to the time to maturity, $\tau$. $L_{t}$ may be viewed as the long-term factor with a loading of 1 , and does not decay to zero in the limit; $S_{t}$ may be interpreted as a short-term factor with a loading that starts at 1 and monotonically decays fast to zero; finally, $C_{t}$ may be viewed as a medium-term factor with a loading that starts at zero, increases and then decays to zero.

Note that long-run interest rates are usually higher than short-run, as an investor is expected to be compensated for funds invested for longer periods. That means that, for most of the periods, $y_{t}$ is an increasing function of the maturity $\tau$. Given that the factor loading $\left(\frac{1-e^{-\lambda \tau}}{\lambda \tau}\right)$ is decreasing with $\tau$, then $S_{t}$ will actually be negative most of the times, and an increase in $S_{t}$ is to be interpreted as a flattening of the curve (with short run interest rates becoming closer to the 
long run rates). This definition of the slope may seem counter-intuitive, but it is a convention in the yield curve literature. ${ }^{1}$

To estimate these components we consider a state-space representation of the model, with a measurement system of equations, in which one relates the yields of different maturities with the three factors:

$$
\left[\begin{array}{c}
y_{t}\left(\tau_{1}\right) \\
y_{t}\left(\tau_{2}\right) \\
\vdots \\
y_{t}\left(\tau_{N}\right)
\end{array}\right]=\left[\begin{array}{ccc}
1 & \left(\frac{1-e^{-\lambda \tau_{1}}}{\lambda \tau_{1}}\right) & \left(\frac{1-e^{-\lambda \tau_{1}}}{\lambda \tau_{1}}-e^{-\lambda \tau_{1}}\right) \\
1 & \left(\frac{1-e^{-\lambda_{2}}}{\lambda \tau_{2}}\right) & \left(\frac{1-e^{-\lambda \tau_{2}}}{\lambda \tau_{2}}-e^{-\lambda \tau_{2}}\right) \\
\vdots & \vdots \\
1 & \left(\frac{1-e^{-\lambda \tau_{3}}}{\lambda \tau_{3}}\right) & \left(\frac{1-e^{-\lambda \tau_{N}}}{\lambda \tau_{N}}-e^{-\lambda \tau_{N}}\right)
\end{array}\right]\left[\begin{array}{c}
L_{t} \\
S_{t} \\
\vdots \\
C_{t}
\end{array}\right]+\left[\begin{array}{c}
\varepsilon_{t}\left(\tau_{1}\right) \\
\varepsilon_{t}\left(\tau_{2}\right) \\
\vdots \\
\varepsilon_{t}\left(\tau_{N}\right)
\end{array}\right],
$$

The factors are modelled as latent factors with the following transition system of equations:

$$
\left[\begin{array}{c}
L_{t}-\bar{L} \\
S_{t}-\bar{S} \\
C_{t}-\bar{C}
\end{array}\right]=\left[\begin{array}{lll}
a_{11} & a_{12} & a_{13} \\
a_{21} & a_{22} & a_{23} \\
a_{31} & a_{32} & a_{33}
\end{array}\right]\left[\begin{array}{c}
L_{t-1}-\bar{L} \\
S_{t-1}-\bar{S} \\
C_{t-1}-\bar{C}
\end{array}\right]+\left[\begin{array}{c}
\eta_{L, t} \\
\eta_{S, t} \\
\eta_{C, t}
\end{array}\right]
$$

where $t=1, \ldots, T$ is the sample period, $\bar{L}, \bar{S}$ and $\bar{C}$ are estimates of the mean values of the three latent factors, and $\eta_{L, t}, \eta_{S, t}$ and $\eta_{C, t}$ are innovations to the autoregressive processes of the latent factors.

We use the Kalman filter to estimate this set of equations. See Aguiar-Conraria et al. (2012) for further details.

\section{$2.3 \quad$ Data}

The daily zero-coupon bond yields for Canada for various maturities — 3, 6, 9, 12, 15, 18, 21, 24, 30, 36, 48, 60, 72, 84, 96, 108 and 120 months - were extracted from the Bank of Canada's website $^{2}$; these yields were converted to monthly yields by a simple average method.

\footnotetext{
${ }^{1}$ Typically, the empirical measure for the slope is: $S l o p e_{t}=y_{t}(3)-y_{t}(120)$. Therefore, a negative value for the slope means that the interest rate is increasing with the maturity.

${ }^{2}$ Data downloaded from http://www.bankofcanada.ca/rates/interest-rates/bond-yield-curves/ on September $22,2016$.
} 
Our choice of macroeconomic variables includes the unemployment rate and CPI Inflation. A business cycle index complements the unemployment rate. While indexes such as GDP growth or output gap are notional measures of the business cycle, their availability in either quarterly or yearly basis make them inappropriate for our purpose. Capacity utilization is another possible indicator, but its unavailability for the entire sample period inhibits its consideration. Following Aguiar-Conraria and Soares (2011), we will rely on the industrial production index's year-on-year growth data.

The Bank of Canada's transition from its previous key monetary instrument - Bank Rate - to the current - Target for the Overnight Rate - on February 22, 1996, creates a bit of a challenge for our choice of monetary policy instrument. Our sample covers the period January 1986 to May 2016. While the data for the Target for the Overnight Rate is unavailable for the entire sample period, the data for Bank Rate is available. Despite this, the correlation between the two rates - Target for the Overnight Rate and Bank Rate - is very strong and this close relation undergirds the choice of the Bank Rate as our monetary policy instrument.

The data for Bank Rate was extracted from the Bank of Canada's website and the data for CPI inflation, and industrial production index were downloaded from the Statistic Canada's website, and IMF, respectively.

The estimated factors, plotted as time plots, and the corresponding wavelet power spectra, are given in Figure 1. In Figure 2 we have the plots and the wavelet power spectra for the macroeconomic activity indicators. ${ }^{3}$

In the plots of the wavelet power, the black conic line identifies the region (usually referred to as the cone-of-influence - COI) where edge effects - unavoidable artifacts appearing when computing the continuous wavelet transform for a finite series - are important; outside this line, the results should be interpreted with caution; see, e.g. Aguiar-Conraria and Soares (2014) for more details. The degree of variability is distinguished by a color spectrum, ranging from dark blue (low variability) to red (high variability). The white lines in the power spectra indicate local

\footnotetext{
${ }^{3}$ There is well-known bias regarding the estimation wavelet coefficients at the lowest scale levels. For that reason we implement the correction proposed by the Liu et al. (2007).
} 
maxima. The black contours signify $5 \%$ significance levels, while the gray contours represent $1 \%$ significance levels. These were computed using a known theoretical distribution for the power, assuming a flat spectrum as the null; see Torrence and Compo (1998) for details.

Latent Factors Plots
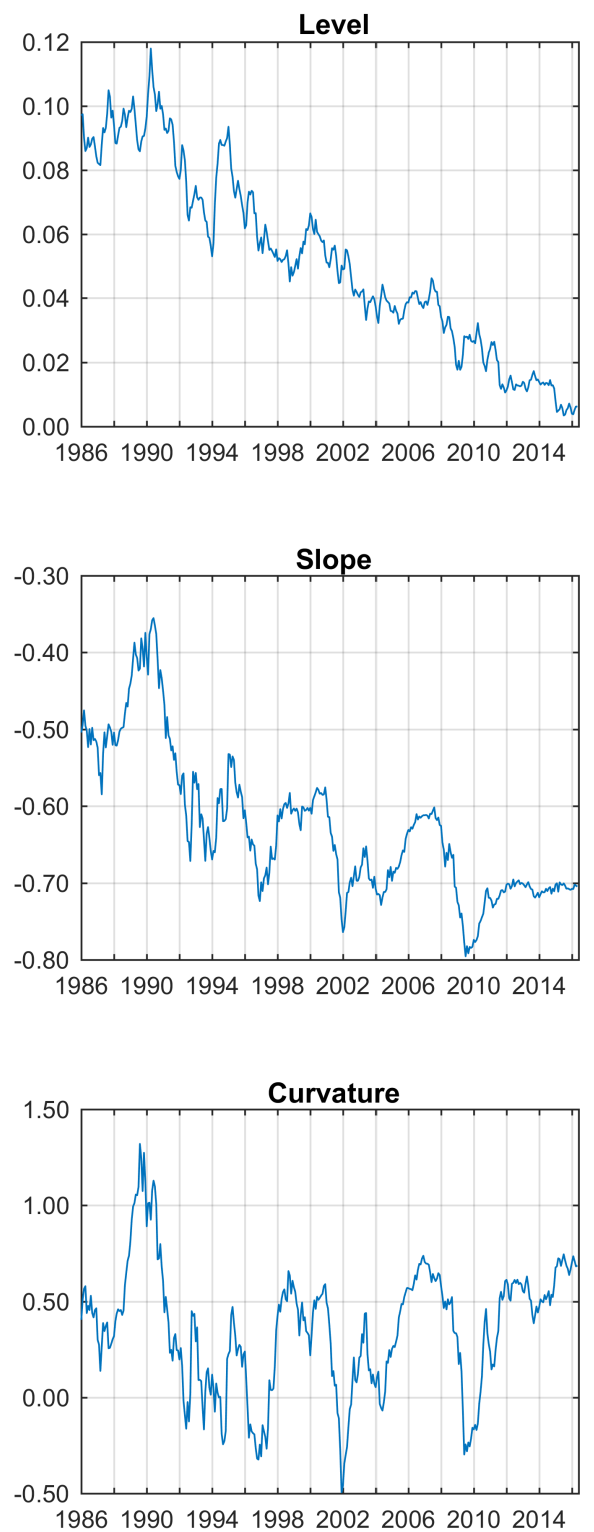

Latent Factors WPS

Level
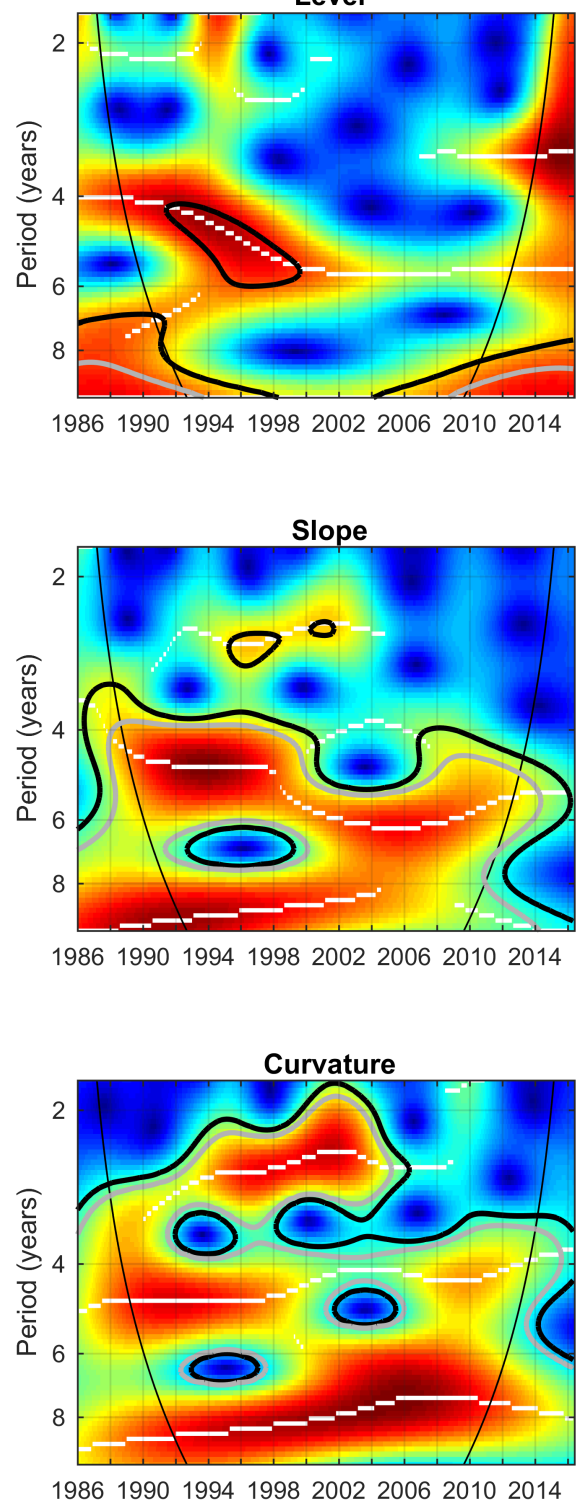

Figure 1: Level, Slope and Curvature of the Canadian yield curve (left panel) and corresponding wavelet power spectra (right panel). The color code for power ranges from dark blue (low power) to red (high power); the black (gray) contours designate the $5 \%$ (1\%) significance levels; the cone of influence, which indicates the region affected by edge effects, is delimited with a black conic line; the white lines show the local maxima of the wavelet power spectra. 

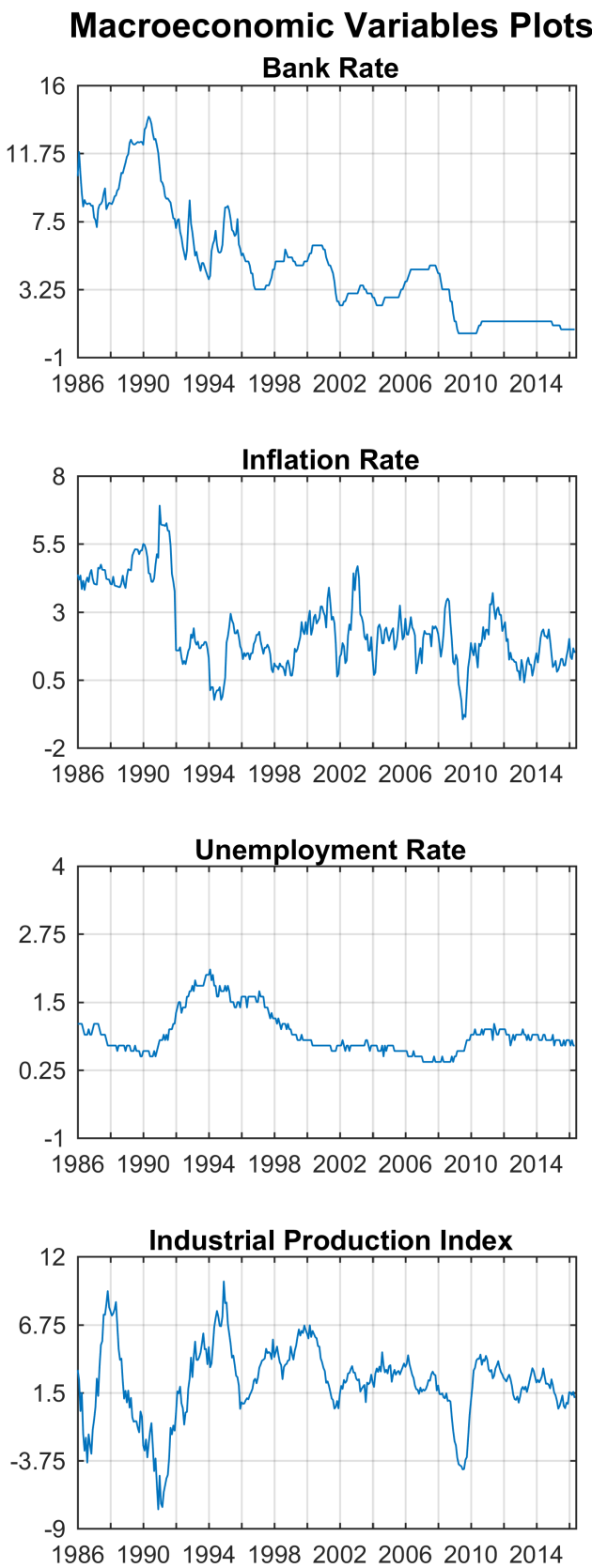

\section{Macoreconomic Variables WPS Bank Rate}

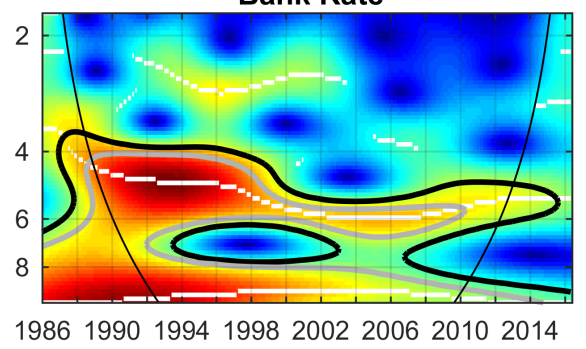

Inflation Rate

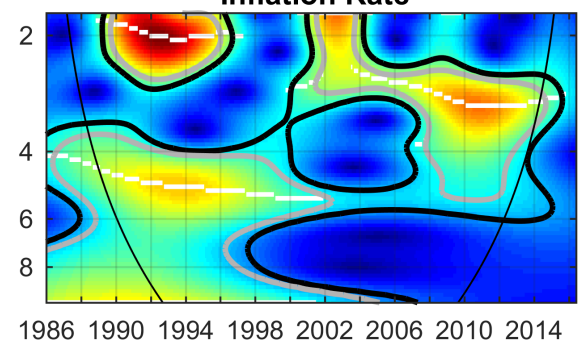

Unemployment Rate

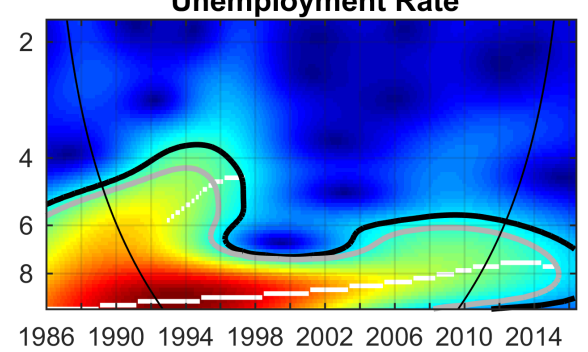

Industrial Production Index

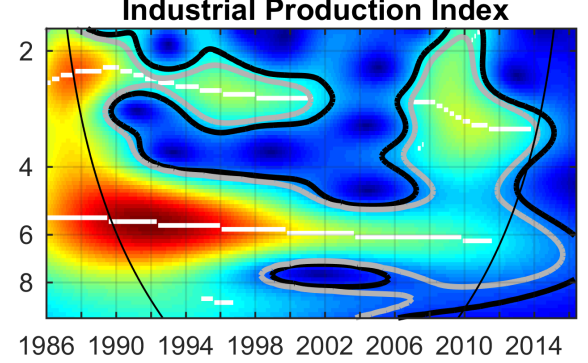

Figure 2: Macroeconomic variables (left panel) and corresponding wavelet power spectra (right panel). The color code for power ranges from dark blue (low power) to red (high power); the black (gray) contours designate the 5\% (1\%) significance levels; the cone of influence, which indicates the region affected by edge effects, is delimited with a black conic line; the white lines show the local maxima of the wavelet power spectra.

For the level factor, one can see that the series was most volatile in the 1990s, in particular for cycles at the lower business cycle frequencies (4 to 8 year period cycles). To be more precise, the volatility is significant at $5 \%$ for the $4 \sim 8$ frequency band, between 1992 and 2000 . If one considers $1 \%$ significance level,then there are almost no significant regions. In the case of the 
slope, if we focus on the $4 \sim 8$ frequency band, the wavelet power spectrum is significant almost across the entire sample. At higher frequencies $(2 \sim 4$ years period cycles) it is also significant in the second half of the 1990s and early 2000s. For the curvature, the high volatility occurs at various frequency bands and also across the entire sample, with several cycles occurring simultaneously, as we can observe a white stripe at a frequency slightly lower that 2 years, another slightly lower than 4 and, finally, another 8 year period cycle. One also observes this multiplicity of cycles for the other factors, but they are not as evident as in the case of the curvature.

It is interesting to note that the bank rate power spectrum is very similar to the power spectrum of the slope (Figure 1): the wavelet power spectrum is significant almost across the entire sample, specially in the $4 \sim 8$ years frequency band.

In the case of the inflation rate, the wavelet power spectrum is quite heterogeneous. One can see a significant island at the 2 years frequency, in the first half of 1990s and then again in the early 2000s. In the first part of the sample, we can also observe some white stripes suggesting that there was an important cycle of 4 to 6 years periodicity, and, approaching the end of the sample, 3 year cycle.

For unemployment, the power spectrum is statistically significant for all the sample period only for lower frequencies.

Finally, for the Industrial Production Index, one observes a very persistent 6 year cycle, although much more important before than after 2000. At higher frequencies, the wavelet power spectrum is also quite high until the year 2000 and then, again around the year 2010.

\section{Empirical Results}

In this section, we present the wavelet coherency and wavelet phase differences between each of the macroeconomic variables and each latent factor of the yield curve and discuss their main implications. Significance tests for the coherency were conducted, based on Monte-Carlo simulations: we fitted an ARMA model to each of the series and constructed new samples with 
the same basic properties; for each pair of series, we performed the exercise 5000 times, and then extracted the critical values at 5 and $1 \%$ significance levels. As for the power, the $5 \%$ significance levels are indicated in the coherency plots with black contours and the $1 \%$ significance levels with gray contours. Because of the possibility of having false positives, in the sense of rejecting the null too often, see Maraun, Kurths and Holschneider (2007), we will focus on the regions which are significant at $1 \%$. The color code for the coherency ranges form dark blue (low coherency) to red (high coherency).

To facilitate the presentation, we display the mean phase difference for two frequency bands — namely for cycles of period $2 \sim 4$ years and cycles of $4 \sim 8$ years. Because the phase differences are measured on a circular scale, the mean is computed as a circular mean, see, e.g. Zar (1998). These (mean) phase differences are indicated in the corresponding plots with a solid black line.

Confidence intervals for the circular mean at each point in time were also computed — we

used the formulas proposed in Zar (1996); see also Berens (2009) — and the interpretation of the mean phase at each point is done considering values as extreme as the two-end points of the corresponding interval. The limits of the confidence intervals for the mean phases are indicated in the pictures with red dashed-lines.

\subsection{Bank Rate and the Yield Curve}

Figure 3 shows the level of coherency between the bank rate and the latent factors (left panel) as well as the phase differences between them (right panel).

The most striking feature of Figure 3 is the high coherency, across most of the times and frequencies, between the bank rate and the slope of the yield curve. Also, the phase difference is basically zero. This means that these two variables are very much synchronized. Much more synchronized than, for example, the level of the yield curve and the bank rate (which are very synchronized only between early 1990s and early 2000s in the $4 \sim 6$ year frequency band).

This implies that when the Bank of Canada changes the bank rate, its main impact is felt 
at the short term interest rates. If it were felt simultaneously for all maturities, then one would expect a much larger statistically significant coherent region between the bank rate and the yield curve level. For example, an increase in the bank rate will lead to a stronger increase at shorter maturities leading to a flattening of the yield curve.

The relation between the curvature and the bank rate is also interesting, as it has a very large region of strong coherency, although, this disappears completely after 2010. The phase difference, slightly negative in several periods, tells us the curvature served as a leading indicator of the bank rate, meaning that changes in the curvature preceded changes in the bank rate in the same direction.
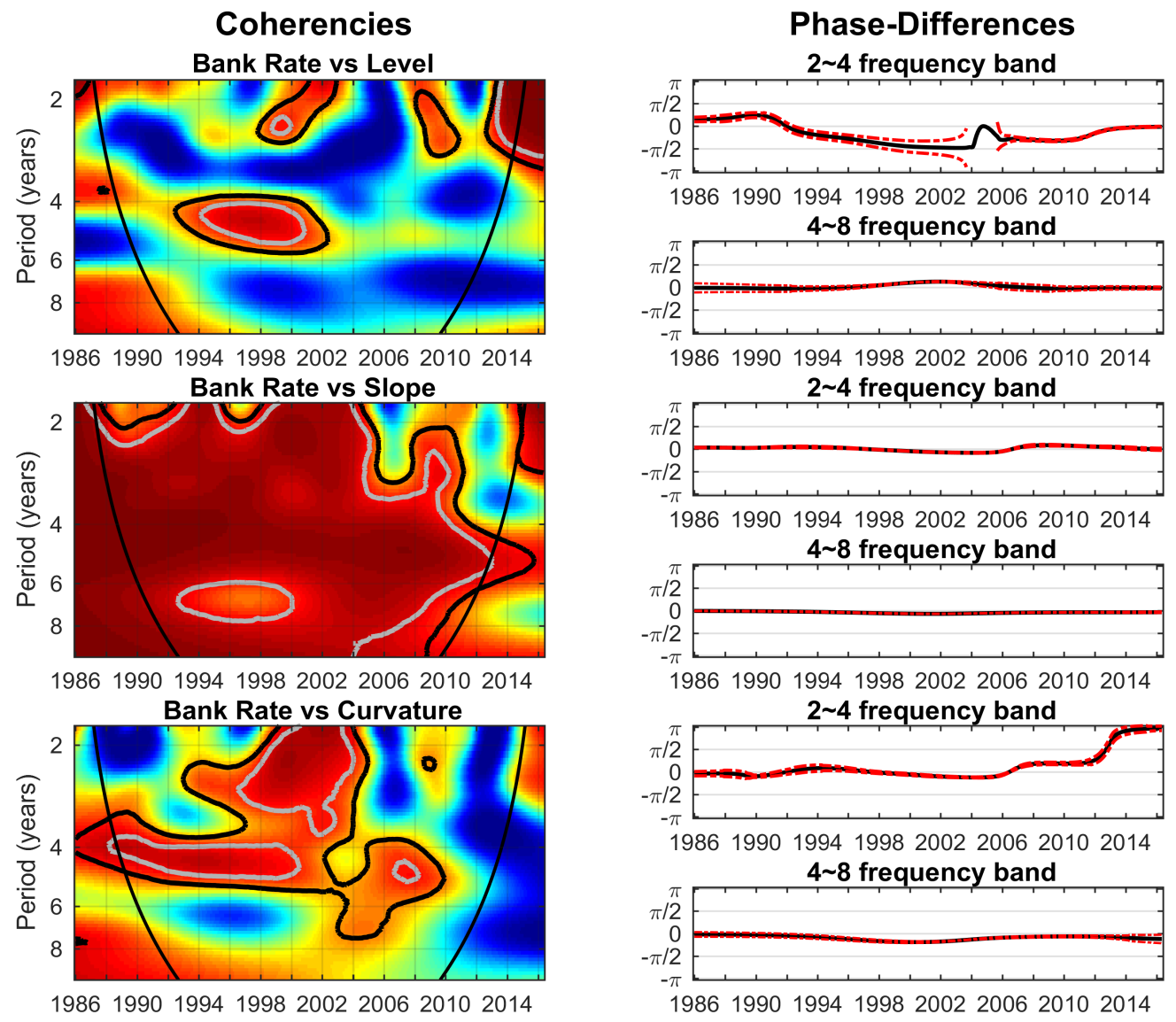

Figure 3: Bank rate and latent factors of yield curve: coherency (left panel) and phase-differences

(right panel). The color code for coherency ranges from dark blue (low coherency) to red (high coherency); the black (gray) contours designate the $5 \%(1 \%)$; the cone of influence, which indicates the region affected by edge effects, is delimited with a black conic line. Phase-differences are indicated with a solid black line and the confidence intervals with a red dashed line. 


\subsection{Inflation Rate and the Yield Curve}

Regarding inflation and the yield curve, Figure 4, we can observe that at 4 to 6 year frequencies and until early 2000s, there is high coherence between the yield curve factors and inflation. The phase differences (in the areas where coherency is statistically significant), consistently between $-\pi / 2$ and zero, tell us that the variables are in-phase with the yield curve factor leading.

If one takes into consideration our results for the bank rate, these results suggest that the Bank of Canada had a very proactive monetary policy. Changes in the slope of the yield curve (and the level between the early 1990s and early 2000s) anticipated changes in the same direction of inflation. This suggests that when the central bank predicted a raise (or fall) in inflation, it would immediately adjust its monetary policy and increase (or decrease) the bank rate.

After that, regions of high coherency are no longer dominant and sometimes the phase difference is between zero and $\pi / 2$ suggesting that monetary policy became more reactive.
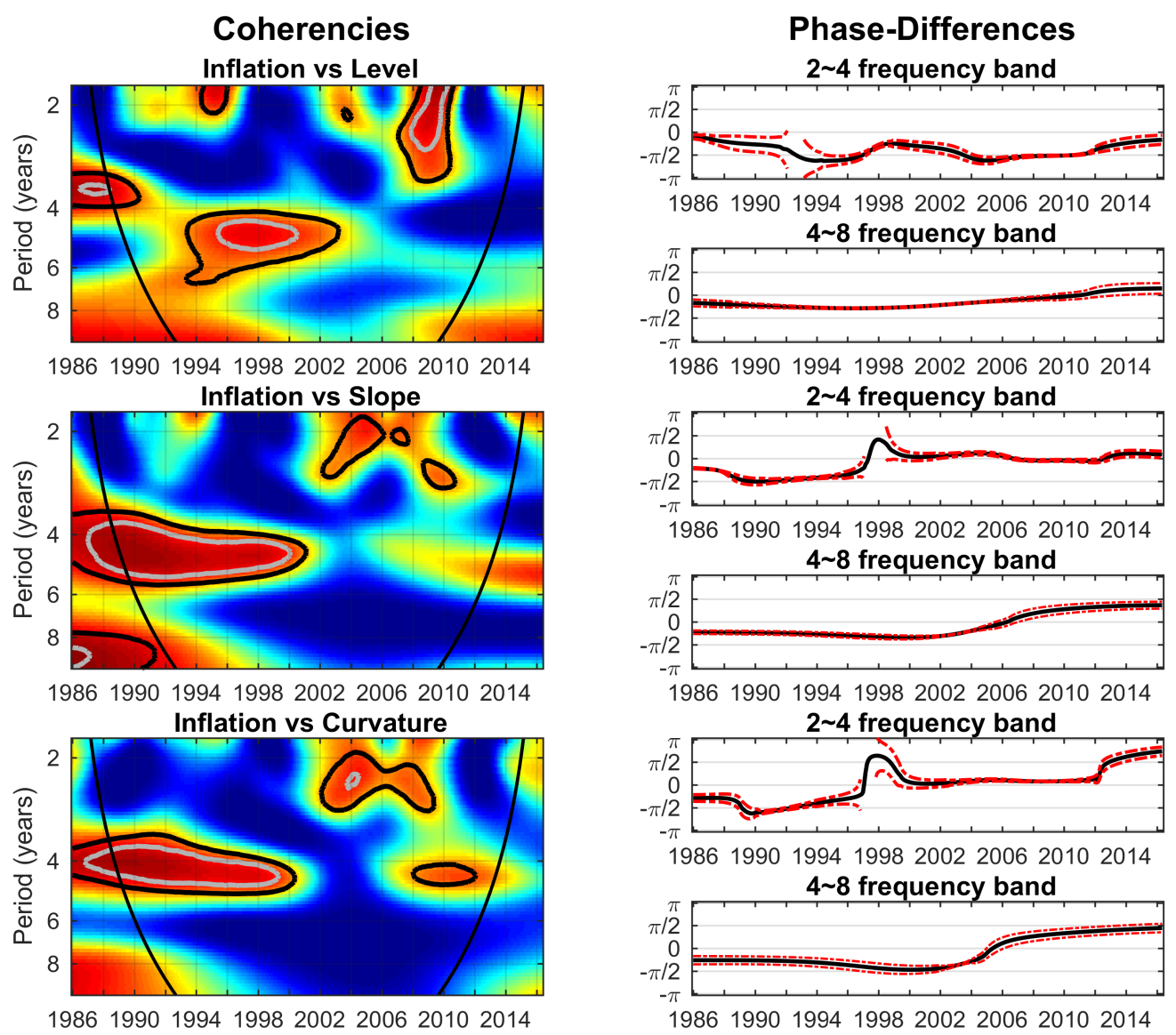

Figure 4: Inflation rate and latent factors of yield curve: coherency (left panel) and phase-differences

(right panel).The color code for coherency ranges from dark blue (low coherency) to red (high 
coherency); the black (gray) contours designate the $5 \%$ (1\%); the cone of influence, which indicates the region affected by edge effects, is delimited with a black conic line. Phase-differences are indicated with a black solid line and the confidence intervals with a red dashed line.

\subsection{Unemployment Rate and the Yield Curve}

Regarding the unemployment rate, one can observe from Figure 5 that the unemployment rate seems to be largely independent of the yield curve level.

This perspective somewhat changes once one looks at the relation with the slope and the curvature, where some regions of statistical significant coherencies can be observed especially for medium and low frequencies until 2010 in the case of the slope, and in the second half of the sample in the case of the curvature, usually with a phase difference indicating an anti-phase relation with the yield curve factor leading.
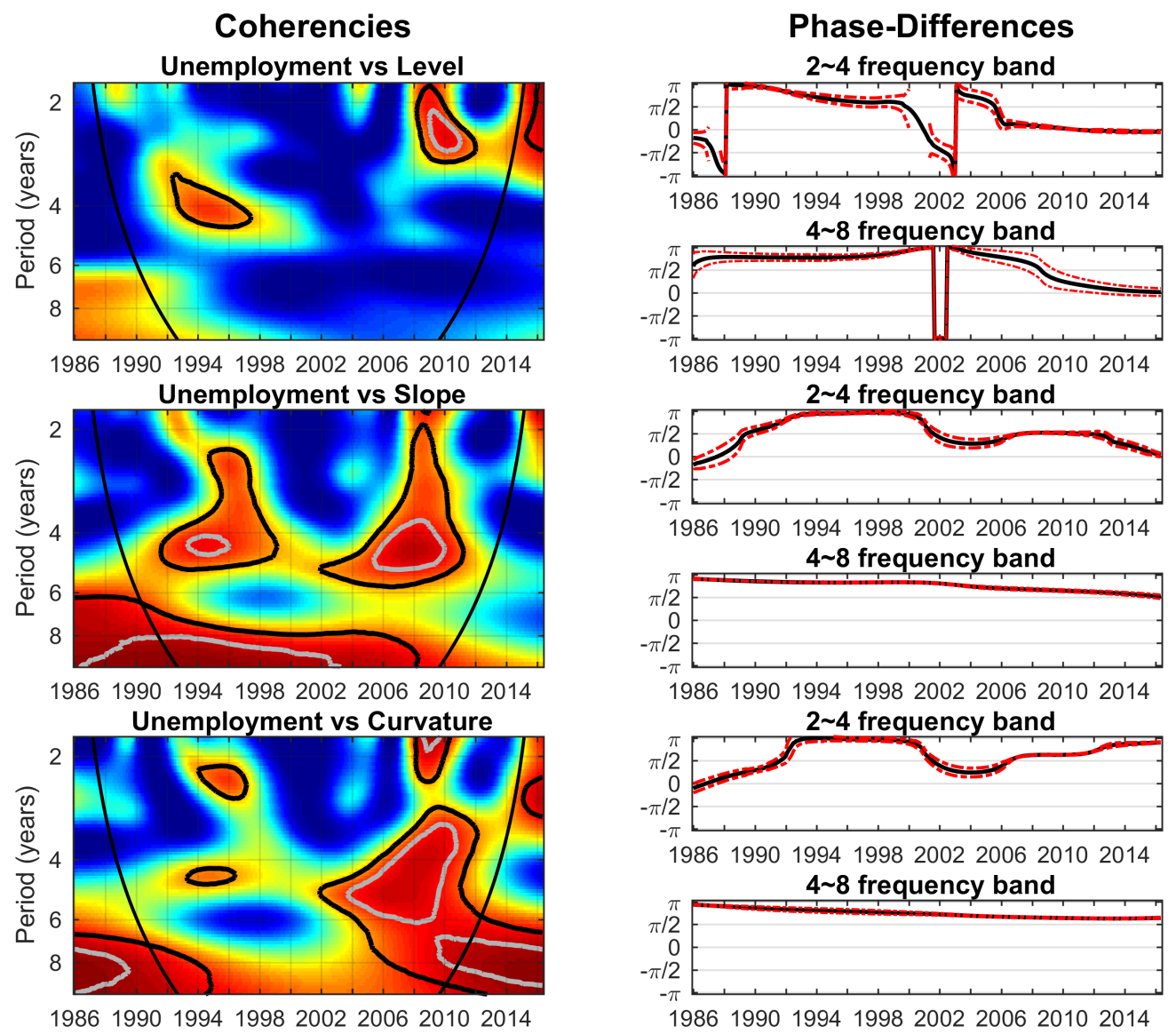

Figure 5: Unemployment rate and latent factors of yield curve: coherency (left panel) and phase-differences (right panel). The color code for coherency ranges from dark blue (low coherency) to red (high coherency); the black (gray) contours designate the 5\% (1\%); the cone of influence, which 
indicates the region affected by edge effects, is delimited with a black conic line. Phase-differences are indicated with a black solid line and the confidence intervals with a red dashed line.

That means that both the slope and the curvature can be seen as leading indicator for the long run evolution of unemployment. Note that wavelet power spectrum of the unemployment rate (Figure 2) tells us that the cyclical behaviour of unemployment occurs mainly at the lower frequencies adding significance to this result.

\subsection{Industrial Production and the Yield Curve}

In this last subsection, we estimate the coherency and phase difference between the Industrial Production and each of the yield curve factors. In Figure 6, we observe several islands of statistically significant coherency.
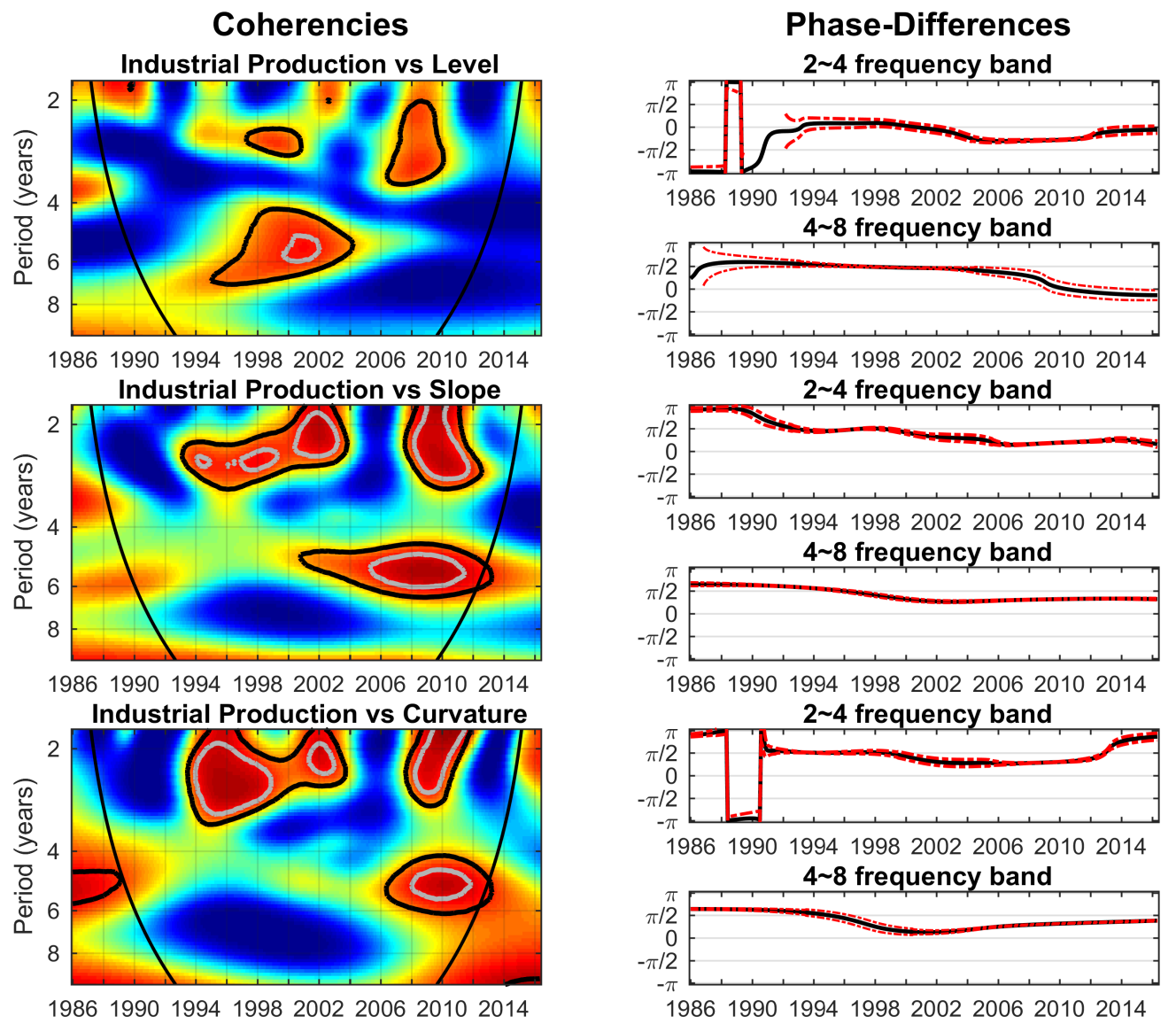

Figure 6: Industrial Production Index and latent factors of yield curve: coherency (left panel) and phase-differences (right panel). The color code for coherency ranges from dark blue (low coherency) to red (high coherency); the black (gray) contours designate the 5\% (1\%); the cone of influence, which 
indicates the region affected by edge effects, is delimited with a black conic line. Phase-differences are indicated with a black solid line and the confidence intervals with a red dashed line.

In the case of the level, the most important region of statistical significance occurs in the $4 \sim 8$ year frequency band between the second half of the 1990s and first half of the 00s (it is the only $5 \%$ significant region that includes a $1 \%$ sub-region). Interestingly, the phase difference is between $\pi / 2$ and $\pi$, suggesting an anti-phase relation with the level of the yield curve leading.

In the case of the slope and the curvature, there are several statistically significant islands, mainly in the $2 \sim 4$ year frequency band, but also for the $4 \sim 6$ years band (towards the end of the sample). For these cases, the phase difference is between $\pi / 2$ and $\pi$, before 1998 , and between 0 and $\pi / 2$ after that. Therefore, these variables are out of phase until 1998, with the yield curve leading, and in-phase after that, with the yield curve factors lagging.

From Figure 3, we know that the slope and curvature are very closely related with our variable representing the monetary policy, the bank rate. Also note that even the level of the yield curve and the bank rate were very synchronized between mid-1990s and early 2000s in the $4 \sim 6$ year frequency band.

In that sense, if we interpret the yield curve factors as a proxies for the monetary policy, the fact that, in Figure 6, the phase differences in the second half of the sample indicate that the Industrial Production Index is in-phase and leading the slope and the curvature, suggests that the Bank of Canada's monetary policy is lagging the evolution of the industrial production.

Given the mandate of the Bank of Canada ( $2 \%$ inflation target), this interpretation is consistent only if the Industrial Production Index is a leading indicator of inflation in the second half of the sample. In the first half, given the anti-phase relation of the industrial production and the yield curve factors, one would also expect the same relation between the industrial production and inflation.

With the aim of testing the predictions in the last paragraphs, we estimate the coherency and phase difference between the Industrial Production and the inflation rate.

In Figure 7, we can observe two main regions of high coherency. One at lower frequencies in the 1990s (only at 5\%), and the other at higher frequencies towards the last third of the sample 
(in this case also at $1 \%$ significance).As expected, the phase difference associated with the first region the phase difference is between $\pi / 2$ and $\pi$, which tells us that there is anti-phase relation with inflation leading and, at higher frequencies (between 2 and 5 year periods) the associated phase difference is between 0 and $\pi / 2$.
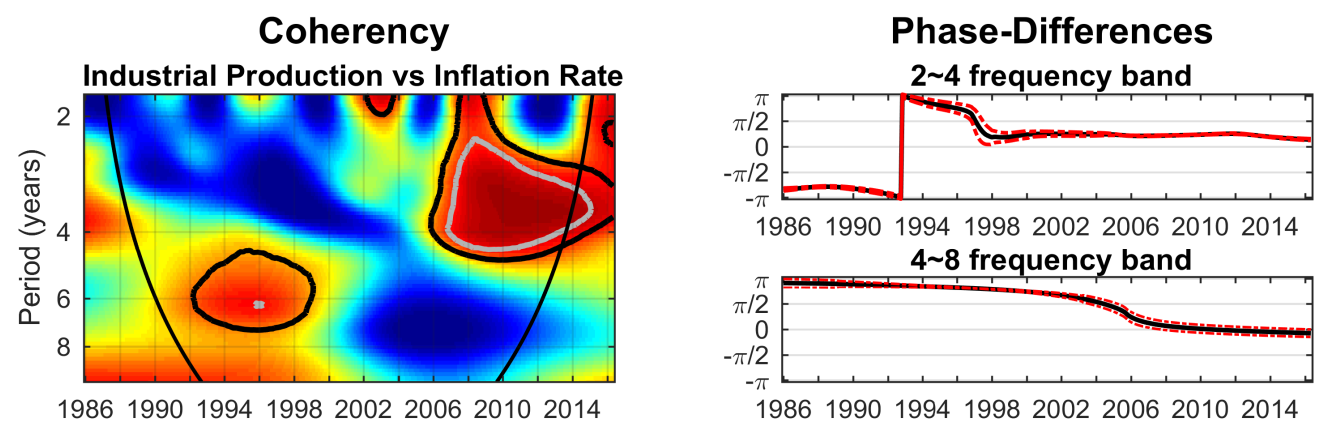

Figure 7: Industrial Production Index and the inflation: coherency (left panel) and phase-differences

(right panel). The color code for coherency ranges from dark blue (low coherency) to red (high coherency); the black (gray) contours designate the 5\% (1\%); the cone of influence, which indicates the region affected by edge effects, is delimited with a black conic line. Phase-differences are indicated with a black solid line and the confidence intervals with a red dashed line.

\section{Conclusion}

In this paper, we used wavelets to study the yield curve and some key macroeconomic indicators, namely the inflation rate, the unemployment rate, the industrial production index and the bank rate (an important monetary policy instrument). Starting with the Continuous Wavelet Transform, we used two important tools: the wavelet coherency and the wavelet phase difference, to study the relation between each of the three-dimensional latent factors of the yield curve and the four macroeconomic indicators.

We reached several conclusions. First, the monetary policy variable, the bank rate, does not have a uniform impact across time horizons. Changes in the bank rate affect, mainly, shortrun interest rates. If one believes that the main driver for economic activity is the long run interest rate (instead of the short run), then it is not difficult to argue that monetary policy is quite ineffective. Second, we concluded that concerning the inflation rate, the Bank of Canada is very proactive, in the sense of trying to act on inflation before it happens. This reiterates 
the information on its website: "monetary policy is always forward looking and the policy rate setting is based on the Bank's judgment of where inflation is likely to be in the future, not what it is today." ${ }^{4}$ Third, regarding the unemployment rate, we found that both the slope and the curvature are leading indicators for the long run evolution of unemployment. Finally, our results suggest that the industrial production index leads the yield curve factors and not the other way. Therefore, nominal interest rates do not seem to be a very important determinant of economic activity.

The main difference between our results and the results of a similar study for the United States (Aguiar-Conraria, Martins and Soares, 2012) is that in the U.S. the Fed interest rates impacted the three factors of the yield curve, showing that monetary policy impacted both short term and long run interest rates. It is also interesting to note that the relations between the yield curve and real macroeconomic activity are stronger in the United States than in Canada.

Our results are not directly comparable with previous studies regarding Canada, because wavelet analysis provide a fundamentally different way of looking at the data. However, it is fair to say that our conclusions align with the existing studies on Canadian yield curve in certain aspects. For instance, our findings on the proactiveness of the Canadian monetary policy is consistent with Lange (2013) on the forward-looking, monetary policy stance on future inflation. Similarly, our result on the link between latent factors and monetary policy rate partly aligns with the existing studies. For example, our result on the high coherency between monetary policy rate and the slope factor share some semblance with the findings of Lange (2013) on the large positive links between monetary policy rate and the same factor (although our results are not as strong as Lange's regarding level factor). To be more precise, our findings show an in-phase relation between the monetary policy rate and the level factor, which is consistent with Lange (2013), but there appears to be a moderate coherency between them in the early 1990s and 2000s. Our results regarding the relation between the yield curve and real activity differ from Lange, who found a very strong relation between them. Even stronger than in the United States, which is contrary to our finds. It is left for future research an attempt to reconcile the

\footnotetext{
${ }^{4}$ http://www.bankofcanada.ca/core-functions/monetary-policy/
} 
results.

\section{Compliance with Ethical Standards}

- The research unit of Aguiar-Conraria and Soares receives funding from COMPETE reference number POCI-01-0145-FEDER-006683, with the FCT/MEC's (Fundação para a Ciência e a Tecnologia, I.P.) financial support through national funding and by the ERDF through the Operational Programme on "Competitiveness and Internationalization - COMPETE 2020 under the PT2020 Partnership Agreement.

- The authors declare that they have no conflict of interest.

- This article does not contain any studies with human participants or animals performed by any of the authors. 


\section{References}

[1] Aguiar-Conraria, L., Magalhães, P.C. and Soares, M. (2012). "Cycles in Politics: Wavelet Analysis of Political Time Series", The American Journal of Political Science, vol. 56, 500-518.

[2] Aguiar-Conraria, L., Magalhães, P.C. and Soares, M. (2013). "The nationalization of electoral cycles in the United States: A wavelet analysis", Public Choice, vol. 156, 387-408.

[3] Aguiar-Conraria, L., Martins, M. M. and Soares, M. J. (2012). "The yield curve and the macro-economy across time and frequencies", Journal of Economic Dynamics and Control, 36(12), 1950-1970.

[4] Aguiar-Conraria, L. and Soares, M. J. (2011). "Business cycle synchronization and the Euro: A wavelet analysis", Journal of Macroeconomics, vol. 33, 477-489.

[5] Aguiar-Conraria, L. and Soares, M. J. (2014). "The continuous wavelet transform: moving beyond uni and bivariate analysis", Journal of Economic Surveys, vol. 28(2), 344-375.

[6] Ang, A. and Piazzesi, M. (2003). "A no-arbitrage vector autoregression of term structure dynamics with macroeconomic and latent variables", Journal of Monetary Economics, vol. $50,745-787$.

[7] Benati, L. and Goodhart, C. (2008). "Investigating time-variation in the marginal predictive power of the yield spread", Journal of Economic Dynamics and Control, vol. 32(4), 12361272.

[8] Berens, P. (2009). "CircStat: A MATLAB Toolbox for Circular Statistics", Journal of Statistical Software, vol. 31(10), 1-21.

[9] Booth, L., Georgopoulos, G. and Hejazi, W. (2007). "What Drives Provincial-Canada Yield Spreads?", Canadian Journal of Economics, vol. 40, 1008-1032. 
[10] Cazelles, B., Chavez, M., de Magny, G. C., Guégan, J-F and Hales, S. (2007). "Timedependent spectral analysis of epidemiological time-series with wavelets", Journal of the Royal Society Interface, vol. 4, 625-636.

[11] Cook, T. and Hahn, T. (1989). "The effect of changes in the Federal funds rate target on market interest rates in the 1970s", Journal of Monetary Economics, vol. 24(9), 331-351.

[12] Dewachter, H. and Lyrio, M. (2006). "Macroeconomic factors in the term structure of interest rates", Journal of Money, Credit and Banking, Vol. 38(1), 119-140.

[13] Diebold, F. X. and Li, C. (2006). "Forecasting the term structure of government bond yields", Journal of Econometrics, 130(2), 337-364.

[14] Diebold, F. X. and Rudebusch, G. D. (2013). Yield Curve Modeling and Forecasting: The Dynamic Nelson-Siegel Approach. Princeton University Press.

[15] Diebold, F. X., Rudebusch, G. D. and Aruoba, S. B. (2006). "The macroeconomy and the yield curve: a dynamic latent factor approach", Journal of Econometrics, vol. 131(1), 309-338.

[16] Edelberg, W. and Marshall, D. E. (1996). "Monetary policy shocks and long-term interest rates", Economic Perspectives, vol. 20, 2-17.

[17] Estrella, A. and Hardouvelis, G.A. (1991). "The term structure as a predictor of real economic activity", Journal of Finance, vol. 46, 555-576.

[18] Evans, C. L. and Marshall, D.E. (2007). "Economic determinants of the mominal treasury yield curve", Journal of Monetary Economics, 54, 1986-2003.

[19] Gallegati, M., Ramsey, J. and Semmler, W. (2014). "Interest rate spreads and output: A time scale decomposition analysis using wavelets", Computational Statistics and Data Analysis, vol. 76, 283-290. 
[20] Garcia, R. and Luger, R. (2007). "The Canadian macroeconomy and the yield curve: an equilibrium based approach?", Canadian Journal of Economics/Revue canadienne d'économique, 40(2), 561-583.

[21] Geiger, F. (2011). The Yield Curve and Financial Risk Premia. Implications for Monetary Policy, Economics Macroeconomics / Monetary Economics / Growth Lecture Notes in Economics and Mathematical Systems, vol. 465, Springer.

[22] Goupillaud, P., Grossman, A. and Morlet, J. (1984). "Cycle-octave and related transforms in seismic signal analysis", Geoexploration, vol. 23, 85-102.

[23] Hao, L. and Ng, E. C. (2011). "Predicting Canadian recessions using dynamic probit modelling approaches", Canadian Journal of Economics/Revue canadienne d'économique, 44(4), 1297-1330.

[24] Hejazi, W., Lai, H. and Yang, X. (2000). The expectations hypothesis, term premia, and the Canadian term structure of interest rates. Canadian Journal of Economics/Revue canadienne d'économique, vol. 33(1), 133-148.

[25] Kozicki, S., and Tinsley, P.A. (2001). "Shifting endpoints in the term structure of interest rates", Journal of Monetary Economics, vol. 47, 613-652.

[26] Lange, R. H. (2013). "The Canadian macroeconomy and the yield curve: A dynamic latent factor approach", International Review of Economics \& Finance, 27, 261-274.

[27] Liu Y., San Liang X., Weisberg R.H. (2007). "Rectification of the bias in the wavelet power spectrum", Journal of Atmospheric and Oceanic Technology, vol. 24, 2093-2102.

[28] Maraun D, Kurths J, Holschneider M (2007). "Nonstationary Gaussian processes in wavelet domain: synthesis, estimation, and significance testing". Physical Review E, vol. 75(1).

[29] Moneta, F. (2005). "Does the yield spread predict recessions in the Euro area?", International Finance, vol. 8(2), 263-301. 
[30] Murray, J. (2013). "Monetary Policy Decision Making at the Bank of Canada." Bank of Canada Review, Autumn, 1-9.

[31] Nelson, C. and A. Siegel (1987). "Parsimonious modeling of yield curves", Journal of Business, vol. 60, 473-489.

[32] Rudebusch, G. D. and Wu, T. (2008). "A macro-finance model of the term structure, monetary policy, and the economy", The Economic Journal, vol. 118, 906-926.

[33] Taylor, J. B. (1993). "Discretion versus policy rules in practice", Carnegie-Rochester Conference Series on Public Policy, vol. 39(1), 195-214

[34] Torrence, C. and Compo, G. (1998). "A practical guide to wavelet analysis", Bulletin of the American Meteorological Society, vol. 79, 61-78.

[35] Wu, T. (2002). "Monetary policy and the slope factors in empirical term structure estimations", Federal Reserve Bank of San Francisco Working Paper 02-07.

[36] Zar, J. H. (1996). Biostatistical Analysis, $3^{\text {rd }}$ Edition, Prentice-Hall. Upper Saddle River, New Jersey. 